\title{
Interacciones entre plantas en un bosque semideciduo micrófilo de Cuba Oriental
}

\author{
Interactions among plants in a coastal dry forest in east Cuba
}

\author{
Arturo Salmerón López ${ }^{\text {a*}}$, Gretel Geada López ${ }^{\text {b }}$ \\ *Autor de correspondencia: ${ }^{a}$ Museo de Historia Natural Tomás Romay, Centro Oriental de Ecosistemas y Biodiversidad, \\ Calle José A. Saco, N. 601, Santiago de Cuba, Cuba, tel.: +53 22 626568, arturo@bioeco.cu \\ ${ }^{\mathrm{b}}$ Universidad de Pinar del Río, Centro de Estudios Forestales, Cuba.
}

\begin{abstract}
SUMMARY
In the microphyll semi-deciduous forest of the Ecological Reserve Siboney Juticí, stress conditions have been conditioned by anthropogenic disturbances, together with climatic and edaphic characteristic. The study develops a method to show the existence of interactions among functional types, species and life class, such as evaluating its magnitude in an unequally disturbed site. The $\chi^{2}$ distribution was applied, considering all possible combinations of species and their life class (seedling, sapling and adult) in the contingence tables. An equation was obtained for an index that made possible to estimate the type (facilitation or competence) and magnitude of the detected interaction. Facilitation and competence interactions were verified. Facilitation was frequent in highly and moderately disturbed sites, and competence in low perturbed sites. Facilitator species correspond fundamentally with the functional type of spreaders and the facilitated were, fundamentally, seedlings and saplings of colonizers species. Some stabilizer species were also documented as facilitators. The facilitator character of some stabilizer species over seedling and sapling of colonizer species in low perturbed sites may be contributing to the stability of these states of the system, in spite of the verified increasing of competence among adult individuals of all functional types in these sites.
\end{abstract}

Key words: facilitation, competence, disturbances, functional types.

\section{RESUMEN}

En el bosque semideciduo micrófilo de la Reserva Ecológica Siboney Juticí, se localizaron sitios con perturbaciones antrópicas que, junto a las condiciones climáticas y edáficas, han determinado condiciones de estrés locales. El estudio desarrolló un método para demostrar la existencia de interacciones entre tipos funcionales, especies y clases de vida y, para evaluar su magnitud, en sitios desigualmente perturbados. Se aplicó la distribución de $\chi^{2}$ considerando todas las combinaciones posibles de las especies presentes y sus clases de vida (plántulas, juveniles y adultos) en las tablas de contingencia. Se obtuvo la ecuación para un índice que permitió estimar el sentido (facilitación o competencia) y la magnitud de la interacción detectada. Se verificó la existencia de interacciones de facilitación y competencia. La facilitación fue más frecuente en los sitios muy y medianamente perturbados y la competencia en los sitios poco perturbados. Las especies facilitadoras se correspondieron fundamentalmente con el tipo funcional cobertoras y las facilitadas fueron fundamentalmente plántulas y juveniles de especies colonizadoras. Algunas especies del tipo estabilizadoras resultaron facilitadoras. El carácter facilitador de algunas especies estabilizadoras sobre plántulas y juveniles de colonizadoras, presentes ambos tipos en los sitios poco perturbados, puede estar aportando estabilidad a esos estados del sistema, a pesar del incremento verificado de la competencia entre los adultos de todos los tipos funcionales en esos sitios.

Palabras clave: facilitación, competencia, perturbaciones, tipos funcionales.

\section{INTRODUCCIÓN}

Para desarrollar estrategias de restauración efectivas debe atenderse a la existencia de interacciones entre plantas. El rol de las interacciones planta - planta como moduladoras de la estructura y de la dinámica de la comunidad vegetal es ampliamente reconocido. Sin la medición de las interacciones entre especies en las comunidades naturales, las predicciones de las dinámicas complejas en estas, serían, en el mejor de los casos, burdas e imperfectas (Armas y Pugnaire 2005, Schiffers y Tielbörger 2006, Maestre et al. 2010, McCluney et al. 2012).

En la vasta literatura disponible sobre interacciones planta - planta se refieren dos tipos básicos: facilitación y competencia (Michalet et al. 2006, Castillo et al. 2010, Soliveres et al. 2011, Gross et al. 2013). El establecimiento de la facilitación como un concepto independiente determina un progreso sustancial en el entendimiento sobre las interacciones en los sistemas ecológicos (Lortie et al. 2004, Brooker y Callaway 2009). 
El rol de la facilitación es más evidenciado en territorios sometidos a elevados niveles de estrés ambiental, tales son los casos de aquellos con determinado nivel de aridez como los ecosistemas mediterráneos, semidesiertos y comunidades vegetales sobre serpentinitas (Michalet et al. 2006, Castillo et al. 2010, Soliveres et al. 2011, Bonanomi et al. 2011, Gross et al. 2013). En las comunidades mediterráneas, se ha llegado a pronosticar procesos de desertificación en ausencia de la facilitación (Kefi et al. 2007, Gross et al. 2013).

Las interacciones de facilitación son definidas como un aspecto clave para el mantenimiento de la biodiversidad y en algunos casos la desaparición de especies nodrizas podría llevar incluso a la pérdida de las especies facilitadas (Castillo y Valiente 2010, Maestre et al. 2010).

Armas y Pugnaire (2005) en comunidades de plantas en zonas semiáridas del sureste español, demuestran que las interacciones gobiernan la dinámica de las poblaciones de dos especies de plantas en esos ambientes y que el tipo de interacción puede cambiar en función de la variabilidad ambiental. Sin embargo, para Maestre et al. (2010) su influencia, particularmente de la facilitación, sobre el funcionamiento de los ecosistemas mediterráneos es menor que la de otros aspectos bióticos, como la cobertura vegetal, que posee un efecto positivo directo.

Armas et al. (2004) proponen un índice relativo para la comparación de las interacciones entre plantas a partir de experiencias manipulativas que denominan RII y que tiene la siguiente ecuación:

$$
\mathrm{RII}=\frac{\mathrm{B}_{\mathrm{w}}-\mathrm{B}_{0}}{\mathrm{~B}_{\mathrm{w}}+\mathrm{B}_{0}}
$$

Donde: $\mathrm{B}_{\mathrm{w}}$ representa las características de la planta (biomasa, crecimiento, etc.) en condiciones de vecindad con otras especies y $\mathrm{B}_{\mathrm{o}}$ representa estas características en condiciones aisladas.

En la ecuación [1], si $\mathrm{B}_{\mathrm{w}}>\mathrm{B}_{\mathrm{o}}$ entonces RII $>1$ y se infiere la existencia de facilitación, en caso contrario se infiere la existencia de competencia.

Otros índices han sido formulados en disímiles condiciones ambientales, pero todos requieren para su determinación el desarrollo de estudios experimentales manipulativos (Brooker et al. 2005, Schiffers y Tielbörger 2006, Brooker y Callaway 2009).

Estudios observacionales en el análisis de las interacciones son considerablemente más escasos y la aproximación más usada para detectar la facilitación es el análisis de las asociaciones espaciales entre especies. Gran parte de estudios se han enfocado a evidenciar la existencia de la facilitación por especies nodrizas, su relación con la riqueza de especies y la diversidad en las comunidades, sin que de alguna manera se mida la magnitud del efecto de la interacción (Drezner 2006, Valiente y Verdu 2008).
Valiente y Verdu (2008) proponen una metodología para evidenciar la existencia de la facilitación, basada en el registro de las especies bajo áreas cubiertas y en espacios abiertos, que utiliza un análisis estadístico de $\chi^{2}$ y determinan, utilizando transeptos en la vegetación, cuánto se diferencia la distribución de facilitadas y facilitadoras de una distribución aleatoria.

La presencia de ambas interacciones, facilitación y competencia, entre diferentes grupos de especies o entre diferentes etapas de la vida de las especies interactuantes, así como la variación en gradientes ambientales en el sentido y la magnitud de las interacciones, están ampliamente documentadas (Michalet el al. 2006, Valiente y Verdú 2008, Maestre et al. 2009, Soliveres et al. 2011). Como promedio, se encuentra que la competencia es mayor en áreas de mayor productividad mientras que la facilitación se incrementa en ambientes secos o de mayor estrés ambiental (Michalet et al. 2006, Schiffers y Tielbörger 2006). Esos resultados contribuyen a la denominada hipótesis del gradiente de estrés (SGH) para la facilitación y la competencia en comunidades de plantas, la cual establece que la frecuencia de interacciones de facilitación y competencia, puede variar a lo largo de gradientes abióticos, en los que la facilitación resulta ser más común en condiciones de elevado estrés (Maestre et al. 2009).

Otros cambios, en este caso temporales, ocurren con el predominio de la facilitación inmediata a la germinación bajo especies nodrizas que cambia a competencia con estas, o entre las mismas especies germinadas, durante el crecimiento. Estos cambios también pueden estar influenciados por fluctuaciones temporales en el ambiente (Schiffers y Tielbörger 2006, Valiente y Verdu 2008, Maestre et al. 2009, Castillo et al. 2010).

Varios autores han documentado la influencia de la relación entre los rasgos funcionales de las especies y las interacciones entre estas, en el proceso de ensamblaje de las comunidades (Armas y Pugnaire 2005, Michalet et al. 2006, Cornwell y Ackerly 2009, Paine et al. 2010, Soliveres et al. 2011). Mediante la evaluación de la variación de los rasgos funcionales se logra considerar las interacciones entre árboles individuales y entre estos y su ambiente (Paine et al. 2010).

De acuerdo con la teoría de la competencia, un incremento en la similitud de los rasgos funcionales conduce a un incremento de este tipo de interacción, entre individuos vecinos (Uriarte et al. 2010, Paine et al. 2010). Uriarte et al. (2010) encuentran que 12 de 18 especies estudiadas aportan información para un modelo según el cual las diferencias entre las especies en cuanto al valor medio de sus rasgos funcionales, estructuran las interacciones entre los individuos vecinos.

Los ambientes en los cuales la facilitación se muestra como un proceso clave (ambientes árticos, alpinos, costeros o áridos) son también particularmente sensibles a los factores antropogénicos que condicionan cambios ambientales tales como el cambio climático, los cambios 
en el uso del suelo y la invasión de especies (Michalet et al. 2006).

Aunque Maestre et al. (2009) consideran como estrés tanto a las tensiones climáticas y edáficas como a las perturbaciones, solo las mencionadas tensiones ambientales (pluviometría, temperaturas, características del suelo, topografía, etc.) son consideradas en la mayoría de los estudios que se realizan a lo largo de gradientes de estrés, mientras que estudios de las interacciones a lo largo de gradientes de perturbación son mucho más escasos (Michalet et al. 2006, Maestre et al. 2009, Soliveres et al. 2012).

La Reserva Ecológica Siboney Juticí, se localiza en una zona de elevado estrés ambiental originado por la escasez de suelo, elevada temperatura y escasas precipitaciones, donde se presenta un bosque semideciduo micrófilo (Reyes y Acosta 2005). En sus ecótopos se localizan sitios calificados como poco, medianamente y muy perturbado $\mathrm{y}$ se definen tres tipos funcionales de respuesta a esas perturbaciones: cobertoras, colonizadoras y estabilizadoras, lo que le confiere una diversidad funcional al bosque que también es modificada por las actividades de origen antrópicos en la reserva (Salmerón et al. 2016a, 2017).

La estructura regenerativa a partir de las proporciones entre adultos, juveniles y plántulas en el bosque semideciduo micrófilo, muestra que el $76,9 \%$ de las especies que conforman los tipos funcionales de respuesta a las perturbaciones en la Reserva Ecológica Siboney Juticí, presentan una regeneración calificada como pobre, muy pobre o simplemente no muestran regeneración y sugiere que las perturbaciones presentes en los sitios muy perturbados o medianamente perturbados (corte de herbáceas para alimentación animal, pastoreo, incendios ocasionales) pueden estar produciendo una erosión de la capacidad de respuesta del sistema boscoso al régimen cambiante de perturbaciones naturales y antrópicas (Salmerón et al. 2016b).

La existencia de áreas perturbadas en los ecótopos de bosque semideciduo micrófilo de la reserva, implica la necesidad de un manejo dirigido a la restauración. Pero la efectividad de esos manejos; aunque se tengan en cuenta la existencia de tipos funcionales, de una determinada diversidad funcional y de la estructura regenerativa, no se logrará completamente si no se tienen en cuenta las interacciones entre los tipos funcionales, las especies y las diferentes clases de vida.

Si se considera la hipótesis del gradiente de estrés para las interacciones, así como que las perturbaciones antrópicas constituyen también una fuente de estrés, entonces podría suponerse que en el bosque semideciduo micrófilo de la Reserva Ecológica Siboney Juticí, existan interacciones tanto de facilitación como competencia que varíen entre los sitios muy, medianamente y poco perturbados.

En este estudio se aspira a desarrollar y aplicar un método que permita tanto demostrar la existencia de la interacción entre plantas, como evaluar la magnitud del efecto debido a estas en sitios desigualmente perturbados, entre tipos funcionales, especies y clases de vida. Se pretende además verificar el posible cambio entre facilitación y competencia al cambiar las clases de vida de las especies.

\section{MÉTODOS}

La Reserva Ecológica Siboney Juticí se encuentra localizada en el borde costero de la región oriental de Cuba, al sureste de Santiago de Cuba. Posee una superficie total de 1.854 ha, de las cuales, aproximadamente 1.050 ha son terrestres ${ }^{1}$. El sector terrestre de la reserva está constituido fundamentalmente por calizas cuaternarias fuertemente carsificadas y escalonadas hacia el Sur en terrazas marinas. Las precipitaciones reportadas en el área no sobrepasan los $800 \mathrm{~mm}$ anuales.

En los lugares más altos de las terrazas, así como en las depresiones, se acumularon depósitos de arcillas resultantes de la decalsificación de las calizas. En esos sitios se estableció un bosque seco reconocido como bosque semideciduo micrófilo (Reyes y Acosta 2005), cuyos ecótopos se extienden por más del $70 \%$ de la superficie terrestre de la Reserva Ecológica. Las especies más abundantes son: Adelia ricinella L., Amyris elemifera L., Banara minutiflora (A. Rich.) Sleumer, Bourreria virgata (Sw.) G. Don. Bursera simaruba (L.) Sargent., Citharexylum spinosum L., Coccoloba diversifolia Jacq., Colubrina elliptica (Sw.) Brizicky et W. L. Stern, Cordia gerascanthus L., Coulteria linnaei (Griseb.) Acev.-Rodr., Crossopetalum rhacoma Crantz, Croton lucidus L., Diospyros grisebachii (Hiern.) Standl., Erithalis fruticosa L., Erythroxylum havanense Jacq., Erythroxylum rotundifolium Lunan, Eugenia cowelli Britton et P. Wilson, Eugenia iteophylla Krug et Urb, Eugenia smonticola (Sw.) DC., Exostema caribaeum (Jacq.) Roem. et Schult., Guettarda cueroensis Britton, Guettarda elliptica Sw., Gymnanthes lucida Sw., Heterosavia bahamensis (Britton) Petra Hoffm., Melochia tomentosa L., Picrodendron baccatum (L.) Krug et Urb. ex Urb., Plumeria obtusa L., Pseudocarpidium avicennioides (A. Rich.) Millsp., Pseudocarpidium ilicifolium (A. Rich.) Millsp., Randia aculeata L., Rauvolfia ligustrina Willd. ex Roem. et Schult., Senna atomaria (L). H. S. Irwin et Barneby, Sideroxylon salicifolium (L.) Lam., Tabebuia myrtifolia (Griseb.) Britton var. myrtifolia, Tecoma stans (L.) Juss. ex Kunth, Thouinia trifoliata Poit., Vachellia macracantha (Humb. et Bonpl. ex Willd.) Seigler et Ebinger, Zanthoxylum fagara (L.) Sarg., Zanthoxylum pistaciifolium Griseb. (Reyes y Acosta 2005).

Verificación de la existencia de interacciones. Se establecieron transeptos en la vegetación como en la metodología de Valiente y Verdu (2008), basada en el registro de las especies bajo áreas cubiertas y en espacios abiertos, que utiliza un análisis estadístico de $\chi^{2}$. En el análisis de los da-

González A, M Abad, A Salmerón, L O Álvarez, M Silot. 2013. Plan de Manejo de la Reserva Ecológica Siboney Juticí, Cuba. BIOECO. 250 p. No publicado. 
tos en las tablas de contingencia correspondientes se realizó una modificación dada por la consideración de todas las combinaciones posibles de las especies presentes y sus clases de vida (plántulas, juveniles y adultos) para las que se analizó su afinidad por espacios cubiertos y descubiertos (cuadro 1). En el estudio se denominó especie diana a aquellas que dominan la cobertura de un parche cubierto, sean documentadas luego como facilitadoras o no. Se consideró a las especies diana como la fuente de la interacción y a las especies sobre las que se analiza el efecto de la interacción se les denominó especies meta.

Considerando que:

$$
\chi_{i j}^{2}=\frac{\left(\mu_{i j}-\varepsilon_{i j}\right)^{2}}{\varepsilon_{i j}}
$$

Donde: $\mu_{\mathrm{ij}}=$ número de elementos de la especie meta i que se observan asociados al parche de la especie dianaj; $\varepsilon_{\mathrm{ij}}=$ número de esos elementos que cabría esperar de acuerdo con la hipótesis nula.

Para definir la existencia de interacción se tuvo en cuenta la significación de los datos en la tabla de contingencia elaborada (cuadro 1), dada por el valor absoluto del residual ajustado (Quinn y Keough 2002):

$$
\mathrm{d}_{\mathrm{ij}}=\frac{\mathrm{e}_{\mathrm{ij}}}{\sqrt{\mathrm{v}_{\mathrm{ij}}}}
$$

Donde:

$$
e_{i j}=\frac{\left(\mu_{i j}-\varepsilon_{i j}\right)}{\sqrt{\varepsilon_{i j}}}
$$

y

$$
\mathrm{v}_{\mathrm{ij}}=\left(1-\frac{\mathrm{n}_{\mathrm{i}}}{\mathrm{N}}\right)\left(1-\frac{\mathrm{n}_{\mathrm{j}}}{\mathrm{N}}\right)
$$

$\mathrm{n}_{\mathrm{i}}=$ número total de individuos en la fila (número total de individuos del mismo estado regenerativo de una especie meta que se encuentran asociados a parches de cualquiera de las especies diana).

$\mathrm{n}_{\mathrm{j}}=$ número total de individuos de todas las especies meta en la columna en la tabla de contingencia (número total de elementos asociado a la especie diana $\mathrm{j}$ ).

$\mathrm{N}=$ número total de individuos.

El residual ajustado $d_{i j}$ permite estimar el nivel de significación independientemente de las frecuencias esperadas y observadas, aunque en todos los casos se tomaron como estrictamente válidos aquellos en que la frecuencia esperada es mayor que uno (en este caso $\varepsilon_{\mathrm{ij}}>1$ ) (Quinn y Keough 2002).

$\mathrm{Si}\left|\mathrm{d}_{\mathrm{ij}}\right| \geq 1,5$ se asumió una desviación significativa de la hipótesis nula atribuible a un determinado tipo de interacción (Quinn y Keough 2002).
Índice para la evaluación de las interacciones. Inicialmente se buscó una ecuación que describiera las posibles variaciones en el número de individuos de las especies meta en función de las variaciones en la superficie de análisis, estuviera o no ocupada por las especies diana. En dicha ecuación se diferenciaron los términos que representan los incrementos esperados en ausencia de la especie diana y los que podrían deberse a la presencia de la especie diana, para aquellos pares de especies en los que se verificó una desviación significativa de la hipótesis nula. Asumiendo los valores y las diferencias entre los números de individuos observados y esperados en cada caso, se obtuvo una ecuación simple que caracteriza los cambios en el número de individuos de las especies meta relacionados con las especies diana.

Evaluación del sentido y la magnitud de la interacción. Para definir si la interacción detectada correspondía a facilitación o a competencia y estimar la magnitud en cada caso, se obtuvo la ecuación para un índice que permitió estimar cuanto se diferencia el incremento (o disminución) en individuos de la especie meta, respecto al incremento (o disminución) esperado debido solo a la variación del área disponible para ella, estuviera previamente ocupada o no esa superficie por una especie diana.

A partir de los análisis precedentes se determinó entre cuáles especies presentan una interacción significativa y, para estas, se aplicó el índice elaborado.

Se realizó el análisis del comportamiento de las interacciones entre las especies correspondientes a los distintos tipos funcionales definidos por Salmerón et al. (2016a).

Se analizaron los cambios ocurridos en el sentido de las perturbaciones para diferentes clases de vida de las especies, cuando estos son verificados, y se compararon para diferentes condiciones de perturbación.

\section{RESULTADOS}

Índice para el balance relativo de las interacciones. Para evaluar el efecto de la interacción en las especies metas cuando se produce un cambio en la superficie del parche cubierto por la especie diana, considerando a la superficie ocupada por la especie diana, se plantea la ecuación:

$$
\mathrm{N}_{\mathrm{m}}\left(\mathrm{s}_{\mathrm{d}}\right)=\mathrm{N}_{\mathrm{m}_{0}}+\psi * \mathrm{~s}_{\mathrm{d}}+\delta
$$

Donde:

$\mathrm{N}_{\mathrm{m}}\left(\mathrm{S}_{\mathrm{d}}\right)=$ número de individuos de la especie meta como función de la superficie $\mathrm{S}_{\mathrm{d}}$ ocupada por la especie diana.

$\mathrm{N}_{\mathrm{n}_{\mathrm{o}}}$ = número de individuos de la especie meta en el resto del espacio no ocupado por la especie diana.

$\Psi=$ incremento esperado específico, de manera tal que:

$$
\psi=\frac{\varepsilon}{S_{\mathrm{dT}}}
$$

Donde: $\mathrm{S}_{\mathrm{dT}}=$ superficie total observada de la especie diana. 


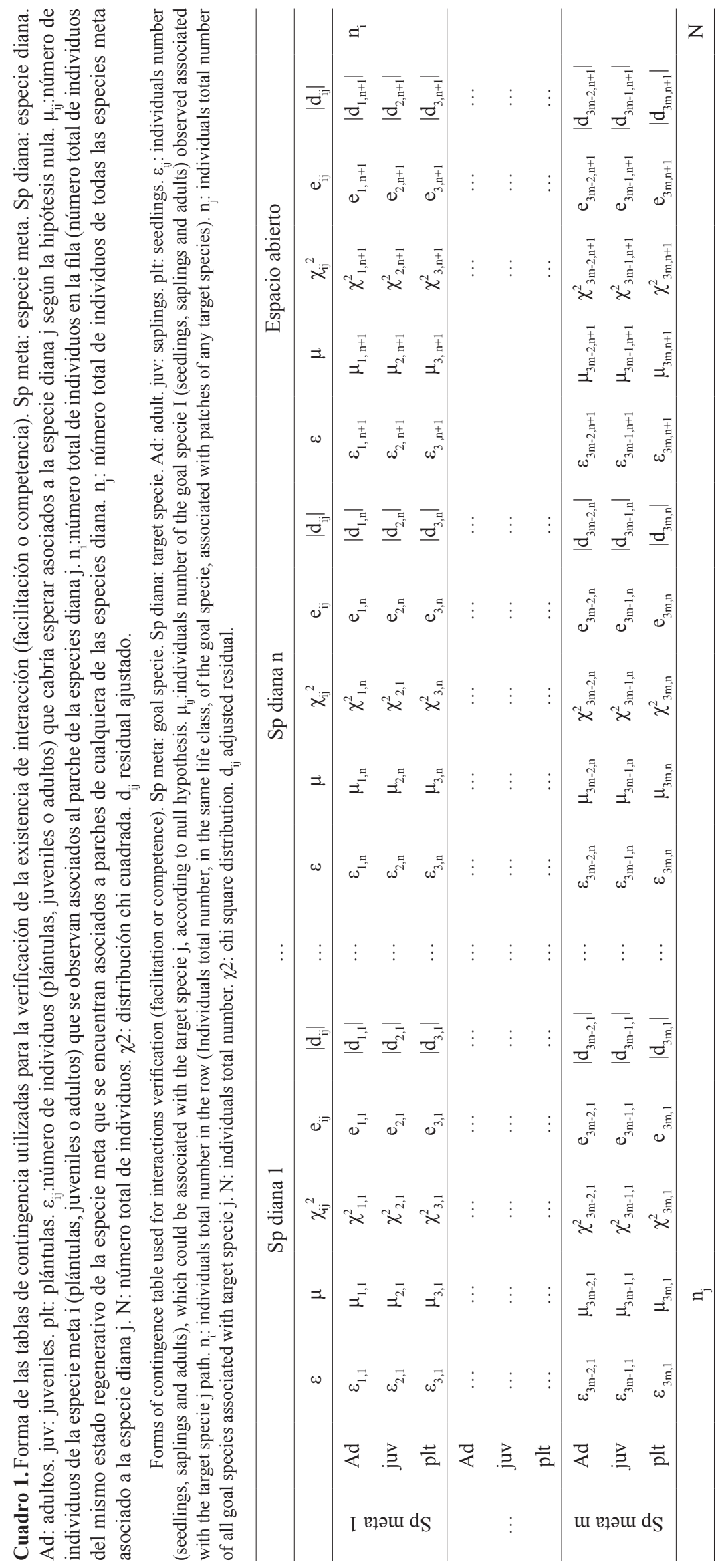


Por lo que en la ecuación [6] el término $\Psi+\mathrm{S}_{\mathrm{d}}$ representa el incremento de individuos de la especie meta, debido solo al aumento de la superficie disponible para la especie meta, independientemente de la presencia de la diana, aunque toma como referencia la superficie de esta.

En la ecuación [6], $\delta$ es la variación del número de individuos de la especie meta debida a la variación de la especie diana, de manera tal que

$$
\delta=f\left(s_{d}\right)=\frac{(\mu-\varepsilon)}{s_{d T}} * s_{d}
$$

o

$$
\delta=\delta_{\mathrm{r}} * \mathrm{~s}_{\mathrm{d}}
$$

Donde:

$$
\delta_{\mathrm{r}}=\frac{(\mu-\varepsilon)}{\mathrm{s}_{\mathrm{dT}}}
$$

Como se observa, la variación de $\mathrm{N}_{\mathrm{m}}\left(\mathrm{S}_{\mathrm{d}}\right)$ debido a $\delta$ si es función de la superficie de la especie diana. Al término $\delta_{\mathrm{r}}$ se le puede denominar "balance relativo de la interacción" y constituye una medida del efecto sobre una especie (especie meta) debido a la presencia de otra (especie diana).

Para el balance relativo de las interacciones $\delta_{\mathrm{r}}$, son posibles los siguientes casos:

$1^{\circ}$. Si no hay interacción y en teoría $\mathrm{N}_{\mathrm{m}}\left(\mathrm{S}_{\mathrm{d}}\right) \mathrm{N}_{\mathrm{n}_{\mathrm{o}}}+\Psi * \mathrm{~S}_{\mathrm{d}}$ $2^{\circ}$. Si $\delta_{\mathrm{r}}$ es positivo, entonces se documenta la facilitación y se incrementa el número de individuos de la especie meta al incrementarse la superficie ocupada por la especie diana según el valor de $\delta_{\mathrm{r}}$.

$3^{\circ}$. Si $\delta_{\mathrm{r}}$ es negativo, entonces se documenta la competencia y disminuye el número de individuos de la especie meta al incrementarse la superficie ocupada por la especie diana según el valor absoluto de $\delta_{\mathrm{r}}$.

En todos los casos se considera que se ha obtenido $\left|d_{i j}\right| \geq 1,5$. En la ecuación [6] cuando $s_{d}=0$, se tiene que $\mathrm{N}_{\mathrm{m}}=\mathrm{N}_{\mathrm{n}_{\mathrm{o}}}$.

Evaluación de las interacciones en el bosque. En los sitios muy perturbados solo se encontraron interacciones de facilitación (cuadro 2).

En los sitios medianamente perturbados, Colubrina elliptica y Tecoma stans, facilitan a los juveniles de Randia aculeata. Ambas especies presentan el mismo $\delta_{\mathrm{r}}$ negativo para las plántulas de esta especie lo que puede sugerir dos posibilidades: O bien las plántulas establecidas de Randia aculeata alcanzan rápidamente el estado de juvenil bajo esas cobertoras (lo que no negaría la facilitación), o bien las primeras se han establecido en espacios muy cercanos y alcanzan el espacio superior a los juveniles o a plántulas preestablecidas de Randia aculeata cubiertas por otra especie no existente en la actualidad. Colubrina elliptica
Cuadro 2. Interacciones en sitios muy perturbados. TF: tipo funcional. Cob: cobertora. Col: colonizadora. Ad: adultos. juv: juveniles. plt: plántulas. $\delta_{\mathrm{r}}$ : balance relativo de las interacciones.

Interactions in highly disturbed sites. TF: functional types.

\begin{tabular}{|c|c|c|c|c|c|}
\hline \multirow{2}{*}{ Especie diana } & \multirow{2}{*}{$\mathrm{TF}$} & \multicolumn{3}{|c|}{ Especie meta } & \multirow{2}{*}{$\delta_{\mathrm{r}}$} \\
\hline & & Especie & Est & $\mathrm{TF}$ & \\
\hline \multirow{3}{*}{$\begin{array}{l}\text { Vachellia } \\
\text { macracantha }\end{array}$} & \multirow{3}{*}{$\mathrm{Cob}$} & Rauwolfia ligustrina & plt & $\mathrm{Col}$ & 0,027 \\
\hline & & Bourreria virgata & plt & $\mathrm{Col}$ & 0,027 \\
\hline & & Randia aculeata & plt & Col & 0,027 \\
\hline
\end{tabular}
Cob: spreaders. Col: colonizers. Ad: adult. juv: saplings. plt: seedlings. $\delta_{\mathrm{r}}$ : relative interactions balance

parece tener comportamientos similares respecto a individuos adultos de las colonizadoras Erythroxylum havanense, Adelia ricinella y Amyris elemifera.

Entre las especies facilitadoras en sitios medianamente perturbados se encuentran también las estabilizadoras Guetarda cueroensis y Pseudocarpidium avicennioides.

Los valores significativos de $\delta_{\mathrm{r}}$ obtenidos en los sitos medianamente perturbados se muestran en el cuadro 3 . En estos sitios predominó la facilitación por especies cobertoras a las especies colonizadoras.

En los sitios poco perturbados se verificó tanto la facilitación como la competencia. En estos sitios Bursera simaruba presenta respecto a Gymnanthes lucida un comportamiento similar a Colubrina elliptica respecto a otras colonizadoras en sitios medianamente perturbados. En estos casos, no se puede hablar con seguridad de facilitación, pues no se ha comprobado ese comportamiento respecto a plántulas o juveniles. Lo más probable es que, como en el caso anterior, Bursera simaruba se establece en espacios abiertos de pequeñas dimensiones cercanos, crece relativamente rápido y cubre a los individuos de Gymnanthes lucida preexistentes, pero esto debe ser comprobado en estudios posteriores. Los valores significativos de $\delta_{\mathrm{r}}$ obtenidos en los sitos poco perturbados se muestran en el cuadro 4

Respecto a posibles cambios en el tipo de interacción, solo pudieron inferirse dos casos. El primero, Randia aculeata presentó $\delta_{\mathrm{r}}$ positivos para plántulas y juveniles bajo varias especies que se han mencionado más arriba, fundamentalmente en sitios medianamente perturbados y alguno en muy perturbados, sin embargo, aunque no hubo $\delta_{\mathrm{r}}$ negativos estadísticamente significativos bajo adultos de otras especies para adultos de Randia aculeata, estos presentaron un $\delta_{\mathrm{r}}$ positivo y significativo estadísticamente para las áreas abiertas en sitios medianamente perturbados lo que puede apuntar a que las plántulas y juveniles se establecen y desarrollan bajo nodrizas pero alcanzan de manera más favorable la adultez cuando el espacio superior queda abierto (salvo en el caso de Senna atomaria, bajo la cual se observó $\delta_{r}$ positivo para adultos en sitios medianamente perturbados). El segundo caso, Gymnanthes lucida 
bajo Picrodendron baccatum, en sitios poco perturbados presentó un $\delta_{\mathrm{r}}$ positivo para las plántulas y negativo para los adultos lo que apunta a un cambio en el sentido de las interacciones entre estas especies.

En el caso de Amyris elemifera, tanto los juveniles como los adultos fueron encontrados siempre bajo nodrizas en sitios medianamente perturbados para adultos bajo Pseudocarpidium avicennioides y bajo Tecoma stans, aunque este último puede ser discutible. Resulta llamativo que para espacios abiertos en sitios medianamente perturbados se encontró para adultos de esta especie $\delta_{\mathrm{r}}=-0,040$. Al parecer, los adultos de Amyris elemifera se establecen y alcanzan la madurez preferentemente bajo alguna nodriza.

\section{DISCUSIÓN}

Para el balance relativo de las interacciones $\delta_{\mathrm{r}}$, son posibles los siguientes casos: $1^{\circ}$. Si $\delta_{\mathrm{r}}$, no hay interacción y en teoría $\mathrm{N}_{\mathrm{m}}\left(\mathrm{S}_{\mathrm{d}}\right) \mathrm{N}_{\mathrm{n}_{\mathrm{o}}}+\Psi * \mathrm{~S}_{\mathrm{d}}$ $2^{\circ}$. Si $\delta_{r}$ es positivo, entonces se documenta la facilitación y se incrementa el número de individuos de la especie meta al incrementarse la superficie ocupada por la especie diana según el valor de $\delta_{\mathrm{r}}$.

$3^{\circ}$. Si $\delta_{\mathrm{r}}$ es negativo, entonces se documenta la competencia y disminuye el número de individuos de la especie meta al incrementarse la superficie ocupada por la especie diana según el valor absoluto de $\delta_{r}$.

En todos los casos se considera que se ha obtenido $\left|\mathrm{d}_{\mathrm{ij}}\right| \geq 1$,5. En la ecuación [6] cuando $\mathrm{s}_{\mathrm{d}}=0$, se tiene que $\mathrm{N}_{\mathrm{m}}=\mathrm{N}_{\mathrm{n}_{0}}$

El índice $\delta_{r}$ permite documentar el cambio de facilitación a competencia, referido en la literatura (Schiffers y Tielbörger 2006, Valiente y Verdu 2008, Maestre et al. 2009, Castillo et al. 2010) cuando en una misma especie diana los valores de $\mathrm{d}_{\mathrm{ij}}$ son significativos para los tres estados de una especie meta, pero los $\delta_{\mathrm{r}}$ son positivos para las

Cuadro 3. Interacciones en sitios medianamente perturbados. TF: Tipo funcional. Cob: cobertora. Col: colonizadora. Est: estabilizadora. Ad: adultos. juv: juveniles. plt: plántulas. $\delta_{\mathrm{r}}$ : balance relativo de las interacciones.

Interactions in moderately disturbed sites. TF: functional types. Cob: spreaders. Col: colonizers. Est: stabilizers. Ad: adult. juv: saplings. plt: seedlings. $\delta_{\mathrm{r}}$ : relative interactions balance.

\begin{tabular}{|c|c|c|c|c|c|}
\hline \multirow{2}{*}{ Especie diana } & \multirow{2}{*}{$\mathrm{TF}$} & \multicolumn{3}{|c|}{ Especie meta } & \multirow{2}{*}{$\delta_{\mathrm{r}}$} \\
\hline & & Especie & $\mathrm{TF}$ & Estado & \\
\hline \multirow{4}{*}{ Vachellia macracantha } & \multirow{4}{*}{ Cob } & \multirow{2}{*}{ Randia aculeata } & \multirow{2}{*}{$\mathrm{Col}$} & plt & 0,049 \\
\hline & & & & juv & 2,811 \\
\hline & & Erythroxylum havanense & $\mathrm{Col}$ & plt & 0,134 \\
\hline & & Guettarda cueroensis & Est & juv & 0,049 \\
\hline \multirow{3}{*}{ Senna atomaria } & \multirow{3}{*}{ Cob } & \multirow{2}{*}{ Randia aculeata } & \multirow{2}{*}{$\mathrm{Col}$} & juv & 0,973 \\
\hline & & & & $\mathrm{Ad}$ & 0,655 \\
\hline & & Erythroxylum havanense & $\mathrm{Col}$ & plt & 0,296 \\
\hline \multirow{6}{*}{ Colubrina elliptica } & \multirow{6}{*}{ Cob } & \multirow{2}{*}{ Randia aculeata } & \multirow{2}{*}{$\mathrm{Col}$} & plt & $-0,295$ \\
\hline & & & & juv & 0,091 \\
\hline & & \multirow{2}{*}{ Erythroxylum havanense } & \multirow{2}{*}{$\mathrm{Col}$} & juv & 0,067 \\
\hline & & & & $\mathrm{Ad}$ & 0,151 \\
\hline & & Adelia ricinella & $\mathrm{Col}$ & $\mathrm{Ad}$ & 0,075 \\
\hline & & Amyris elemifera & $\mathrm{Col}$ & Ad & 0,151 \\
\hline \multirow{2}{*}{ Tecoma stans } & \multirow{2}{*}{ Cob } & \multirow{2}{*}{ Randia aculeata } & \multirow{2}{*}{$\mathrm{Col}$} & plt & $-0,295$ \\
\hline & & & & juv & 2,957 \\
\hline \multirow{2}{*}{ Randia aculeata } & \multirow{2}{*}{$\mathrm{Col}$} & \multirow{2}{*}{ Randia aculeata } & \multirow{2}{*}{$\mathrm{Col}$} & plt & 0,630 \\
\hline & & & & juv & 0,273 \\
\hline \multirow{3}{*}{ Guettarda cueroensis } & \multirow{3}{*}{ Est } & Randia aculeata & $\mathrm{Col}$ & plt & 0,876 \\
\hline & & \multirow{2}{*}{ Erythroxylum havanense } & \multirow{2}{*}{$\mathrm{Col}$} & plt & 0,296 \\
\hline & & & & juv & 0,180 \\
\hline \multirow{2}{*}{ Pseudocarpidium avicennioides } & \multirow{2}{*}{ Est } & \multirow{2}{*}{ Randia aculeata } & \multirow{2}{*}{$\mathrm{Col}$} & plt & 0,529 \\
\hline & & & & juv & 0,576 \\
\hline
\end{tabular}


Cuadro 4. Interacciones en sitios poco perturbados. TF: tipo funcional. Cob: cobertora. Col: colonizadora. Est: estabilizadora. Ad: adultos. juv: juveniles. plt: plántulas. $\delta_{\mathrm{r}}$ : balance relativo de las interacciones.

Interactions in low disturbed sites. TF: functional types. Cob: spreaders. Col: colonizers. Est: stabilizers. Ad: adult. juv: saplings. plt: seedlings. $\delta_{r}$ : relative interactions balance.

\begin{tabular}{|c|c|c|c|c|c|}
\hline \multirow{2}{*}{ Especie diana } & \multirow{2}{*}{$\mathrm{TF}$} & \multicolumn{3}{|c|}{ Especie meta } & \multirow{2}{*}{$\delta_{\mathrm{r}}$} \\
\hline & & Especie & $\mathrm{TF}$ & Estado & \\
\hline \multirow{3}{*}{ Vachellia macracantha } & \multirow{3}{*}{$\mathrm{Cob}$} & Diospyros grisebachii & Col & plt & 0,439 \\
\hline & & \multirow{2}{*}{ Gymnanthes lucida } & \multirow{2}{*}{$\mathrm{Col}$} & plt & 0,797 \\
\hline & & & & juv & 0,861 \\
\hline Bursera simaruba & $\mathrm{Cob}$ & Gymnanthes lucida & $\mathrm{Col}$ & $\mathrm{Ad}$ & 0,795 \\
\hline \multirow{4}{*}{ Picrodendron baccatum } & \multirow{4}{*}{ Est } & \multirow{2}{*}{ Gymnanthes lucida } & \multirow{2}{*}{$\mathrm{Col}$} & plt & 0,095 \\
\hline & & & & Ad & $-0,301$ \\
\hline & & Erythroxylum rotundifolium & Est & plt & 0,063 \\
\hline & & Coccoloba diversifolia & Col & plt & 0,063 \\
\hline Crossopetalum rhacoma & Est & Gymnanthes lucida & $\mathrm{Col}$ & juv & 0,076 \\
\hline \multirow{4}{*}{ Coccoloba diversifolia } & \multirow{4}{*}{$\mathrm{Col}$} & Gymnanthes lucida & Col & plt & $-0,048$ \\
\hline & & Heterosavia bahamensis & $\mathrm{Col}$ & Ad & $-0,232$ \\
\hline & & Picrodendron baccatum & Est & Ad & $-0,072$ \\
\hline & & Bursera simaruba & $\mathrm{Cob}$ & Ad & $-0,064$ \\
\hline Heterosavia bahamensis & Col & Gymnanthes lucida & Col & Ad & $-0,630$ \\
\hline Sideroxylon salicifolium & $\mathrm{Col}$ & Gymnanthes lucida & Col & Ad & $-0,872$ \\
\hline Erithalis fruticosa & Est & Gymnanthes lucida & Col & Ad & $-0,872$ \\
\hline Banara minutiflora & Est & Gymnanthes lucida & Col & Ad & $-0,872$ \\
\hline
\end{tabular}

plántulas o juveniles de la especie meta y negativos para los individuos adultos de la misma, en cuyo caso se interpreta que la nodriza facilita el establecimiento de plántulas y juveniles pero dificulta que estos se conviertan en adultos.

La facilitación por parte de especies nodrizas, fundamentalmente cobertoras, sobre plántulas o juveniles de otras especies, aunque se evidencia también en sitios poco perturbados es más frecuente en este estudio en los sitios medianamente y muy perturbados. Si se tiene en cuenta que al nivel de estrés contribuye también el nivel de perturbación (Maestre et al. 2009), se deben considerar los sitios medianamente y muy perturbados como de mayor estrés, lo que hace coincidir los resultados aquí presentados con los de la mayoría de los autores que se han mencionado en este sentido (Michalet et al. 2006, Castillo et al. 2010, Soliveres et al. 2011, Gross et al. 2013). Al pasar de sitios medianamente perturbados a poco perturbados, la facilitación se mantiene entre algunas especies y comienza a aparecer la competencia, haciéndose esta más frecuente en estos sitios, de manera coincidente con la denominada hipótesis del gradiente de estrés (SGH), (Michalet et al. 2006, Valiente y Verdu 2008, Maestre et al. 2009, Soliveres et al. 2011).
La baja regeneración en los sitios medianamente y muy perturbados, debida probablemente a la incidencia del pastoreo (Salmerón et al. 2016b), es un factor que puede estar dificultando el conocimiento real de los procesos de facilitación y su verdadero rol en la recuperación de este sistema frente al resto de las perturbaciones presentes. Otras especies podrían estar sujetas a la facilitación, pero la ausencia de plántulas o juveniles imposibilita verificarla. En el futuro deben realizarse estudios experimentales en ausencia de pastoreo para precisar la incidencia de la facilitación en la dinámica del bosque semideciduo micrófilo y los mecanismos mediante los cuales esta se produce.

La facilitación es más común entre especies correspondientes a diferentes tipos funcionales y parece ser más demandada por especies de las denominadas colonizadoras (Salmerón et al. 2016a). En los sitios muy perturbados las facilitadoras Vachellia macracantha y Senna atomaria son ambas cobertoras y facilitan a especies colonizadoras aunque estas últimas varían en sitios poco perturbados respecto a los muy y medianamente perturbados. De las nueve especies estadísticamente comprobadas como facilitadas, siete son colonizadoras Rauvolfia ligustrina, Bourreria virgata, Randia aculeata, Erythroxylum havanense, 
Diospyros grisebachii, Gymnanthes lucida y Coccoloba diversifolia.

De las especies estabilizadoras son identificadas como facilitadoras Picrodendron baccatum, Guetarda cueroensis y Pseudocarpidium avicennioides.

Los resultados de este estudio parecen coincidir con los de Uriarte et al. (2010) y Paine et al. (2010) en cuanto a que las características funcionales de las especies condicionan las interacciones entre estas.

En los sitios poco perturbados predominan las interacciones de tipo competitivo. Esta se comprueba estadísticamente para adultos de las especies Coccoloba diversifolia, Gymnanthes lucida, Heterosavia bahamensis, Sideroxylon salicifolium, todas colonizadoras. No obstante, se verifica la competencia entre la colonizadora Gymnanthes lucida y las estabilizadoras Erithalis fruticosa, Picrodendron baccatum y Banara minutiflora. Datos obtenidos respecto a la demanda de espacios abiertos para adultos de 14 especies en sitios poco perturbados, aunque no entran en el objetivo del presente estudio constituyen además un elemento que apunta a que en estos sitios los adultos tienden a competir por el espacio y la luz.

Por su parte, Maestre et al. (2010) reconoce que la cobertura tiene más influencia sobre el funcionamiento del sistema que las interacciones. No obstante, si se considera que la presencia de plantas nodrizas aisladas es un elemento clave en la estructura y dinámica de los ecosistemas, lo que ha sido validado además en varios estudios (Valiente y Verdu 2008, Maestre et al. 2010) entonces resulta evidente que las interacciones positivas influyen de manera directa en el incremento de la cobertura vegetal y en el funcionamiento de estos ecosistemas, particularmente desde el punto de vista que se analiza en este estudio. Al menos puede afirmarse que la facilitación y el incremento de algunas especies cobertoras son inherentes una a la otra, este proceso favorece la aparición de determinadas especies colonizadoras y esto, a su vez, favorece el avance del sistema a estados de mayor integridad, con la aparición de las especies estabilizadoras en los estados poco perturbados.

De acuerdo con el enfoque que se sigue en este estudio, lo expuesto por Maestre et al. (2010) respecto a la mayor importancia de la cobertura vegetal para el funcionamiento de los ecosistemas, es una evidencia de que en el mantenimiento de la integridad de los ecosistemas, tanto la composición como la estructura y las interacciones, son importantes y que al alterar uno de sus atributos se alteran los otros. Maestre et al. (2010) al alterar la cobertura se está alterando la composición, esto implica ya una alteración de estructura y por tanto de las interacciones. En el presente estudio, a partir de los análisis realizados, no se considera más importante un atributo (de composición o de estructura) que otro de interacciones. Los elementos funcionales del sistema (desde el punto de vista funcional de que se trate) están profundamente interconectados y no se puede (al menos resultaría muy difícil) alterar algunos manteniendo constantes los otros.
El estudio de las interacciones en gradientes de perturbación al interior de sistemas con elevado estrés ambiental (climático y edáfico fundamentalmente) debe ser priorizado en el futuro inmediato si se tiene en cuenta que, como se ha advertido, son particularmente sensibles a los factores antropogénicos que condicionan cambios ambientales, tales como los cambios en el uso del suelo y la invasión de especies (Michalet et al. 2006).

\section{CONCLUSIONES}

En los sitios sometidos a diferentes niveles de perturbación en el bosque semideciduo micrófilo en la Reserva Ecológica Siboney Juticí se verifica la existencia de interacciones tanto de facilitación como de competencia. La facilitación es más frecuente en los sitios muy y medianamente perturbados. La competencia es más frecuente en los sitios poco perturbados.

El índice propuesto (balance relativo de las interacciones) y el método para su evaluación, permiten no solo identificar la existencia de facilitación, sino que posibilita definir la existencia de competencia y estimar la magnitud de ambas interacciones entre especies, tipos funcionales y estados de vida.

La facilitación es más frecuente entre especies correspondientes a diferentes tipos funcionales y resulta más demandada por las plántulas y juveniles de las especies colonizadoras. Las especies facilitadoras son fundamentalmente las cobertoras y algunas estabilizadoras.

El carácter facilitador de algunas especies estabilizadoras sobre plántulas y juveniles de colonizadoras, en los sitios poco perturbados, puede estar aportando estabilidad a esos estados del sistema, a pesar del incremento verificado de la competencia, en esos sitios, entre los adultos cualesquiera sean sus tipos funcionales.

\section{REFERENCIAS}

Armas C, FI Pugnaire. 2005. Plant interactions govern population dynamics in a semi-arid plant community. Journal of Ecology 93: 978-989.

Armas C, R Ordiales, F Pugnaire. 2004. Measuring plant interactions: a new comparative index. Ecology 85(10): 26822686.

Bonanomi G, G Incerti, S Mazzoleni. 2011. Assessing occurrence, specificity, and mechanisms of plant facilitation in terrestrial ecosystems. Plant Ecology 212:1777-1790.

Brooker RW, RM Callaway. 2009. Facilitation in the conceptual melting pot. Journal of Ecology 97: 1117-1120.

Brooker R, Z Kikvidze, FI Pugnaire, RM Callaway, P Choler, ChJ Lortie, R Michalet. 2005. The importance of importance. Oikos 109: 63 -70.

Castillo JP, M Verdu, A Valiente. 2010. Neighborhood phylodiversity affects plant performance. Ecology 91(12): 36563663.

Cornwell WK, A Ackerly. 2009. Community assembly and shifts in plant trait distributions across an environmental gradient in coastal California. Ecological Monographs 79(1): 109-126. 
Drezner TD. 2006. Plant facilitation in extreme environments: The non-random distribution of saguaro cacti (Carnegiea gigantea) under their nurse associates and the relationship to nurse architecture. Journal of Arid Environments 65: 46-61.

Gross N, L Borger, SI Soriano, YL Bagousse, JL Quero, M García, E Valencia, FT Maestre. 2013. Uncovering multiscale effects of aridity and biotic interactions on the functional structure of Mediterranean shrublands. Journal of Ecology 101: 637-649.

Lortie Ch J, RW Brooker, P Choler, Z Kikvidze, R Michalet, FI Pugnaire, RM Callaway. 2004. Rethinking plant community theory. Oikos 107(2): 433-438.

Maestre FT, MA Bowker, C Escolar, MD Puche, S Soliveres, S Maltez-Mouro, P García-Palacios, AP Castillo-Monroy, I Martínez, A Escudero. 2010. Do biotic interactions modulate ecosystem functioning along stress gradients? Insights from semi-arid plant and biological soil crust communities. Philosophical Transactions of Royal Society B 365: 20572070.

Maestre FT, RM Callaway, F Valladares, Ch J Lortie. 2009. Refining the stress-gradient hypothesis for competition and facilitation in plant communities. Journal of Ecology 97: 199-205.

McCluney KE, J Belnap, SL Collins, AL González, EM Hagen, JN Holland, BP Kotler, FT Maestre, SD Smith, BO Wolf. 2012. Biological Reviews 87: 563-582. DOI: 10.1111/j.1469-185X.2011.00209.x.

Michalet R, RW Brooker, LA Cavieres. 2006. Do biotic interactions shape both sides of the humped-back model of species richness in plant communities? Ecology Letters 9: 767-773.

Paine CET, Ch Baraloto, J Chave, B Hérault. 2010. Functional traits of individual trees reveal ecological constraints on community assembly in tropical rain forests. Oikos 120: 720-727. DOI: 10.1111/j.1600-0706.2010.19110.x.

Quinn GP, MJ Keough. 2002. Experimental Design and Data Analysis for Biologists. Cambridge, UK. Cambridge University Press. 557 p.

Reyes J, F Acosta. 2005. Vegetación Terrestre en Cuba: Siboney-
Juticí. Rapid Biological Inventories, Report 10. Chicago, USA. The Field Museum. 73 p.

Salmerón A, A González, G Geada. 2016a. Tipos funcionales de plantas según su respuesta a las perturbaciones en un bosque semideciduo micrófilo costero de Cuba Oriental. Bosque 37(1): 131-141. DOI: 10.4067/S071792002016000100013.

Salmerón A, G Geada, LO Alvarez. 2016b. Estructura regenerativa funcional en el bosque semideciduo micrófilo de la reserva ecológica Siboney Juticí. Revista Forestal Baracoa 35(2): 83-91.

Salmerón A, G Geada, MC Fagilde. 2017. Propuesta de un índice de diversidad funcional. Aplicación a un bosque semideciduo micrófilo de Cuba Oriental. Bosque 38(3): 457-466. DOI: $10.4067 / \mathrm{S} 0717-92002017000300003$.

Schiffers K, K Tielbörger. 2006. Ontogenetic shifts in interactions among annual plants. Journal of Ecology 94: 336-341. DOI: 10.1111/j.1365-745.2006.01097.x.

Soliveres S, JD Eldridge, F Hemmings, FT Maestre. 2012. Nurse plant effects on plant species richness in drylands: The role of grazing, rainfall and species specificity. Perspectives in Plant Ecology, Evolution and Systematics 14(2012): 402441.

Soliveres S, JD Eldridge, FT Maestre, MA Bowker, M Tighed, A Escudero. 2011. Microhabitat amelioration and reduced competition among understorey plants as drivers of facilitation across environmental gradients: Towards a unifying framework. Perspectives in Plant Ecology, Evolution and Systematics 13: 247-258.

Uriarte M, NG Swenson, RL Chazdon, LS Comita, WJ Kress, D Erickson, J Forero- Montan, JK Zimmerman, J Thompson. 2010. Trait similarity, shared ancestry and the structure of neighbourhood interactions in a subtropical wet forest: implications for community assembly. Ecology Letters 13: 1503-1514.

Valiente A, M Verdu. 2008. Temporal shifts from facilitation to competition occur between closely related taxa. Journal of Ecology 96: 489-494. 